\title{
Novel WLAN-Based Indoor Device-Free Motion Detection System Using PHY Layer Information
}

\author{
Dr.S.KANNAN \\ Professor \\ Information Technology \\ E.G.S. Pillay Engineering College, \\ Nagapattinam - 611002. Tamilnadu,India. \\ kannan.pot@gmail.com
}

\author{
R.ROGER SAMSON \\ Assistant Professor \\ Computer Science and Engineering \\ Anjalai Ammal-Mahalingam Engineering \\ College, Koyilvenni-614403. Tamilnadu,India. \\ jacksonjonah1986@gmail.com
}

\begin{abstract}
With the quick advancement of WLAN innovation, remote gadget free latent human location turns into a recently creating system and holds extra potential to worldwide and inescapable shrewd applications. As of late, indoor fine-grained gadget free latent human movement location bolstered the PHY layer information is immediately created. Past remote gadget free aloof human identification frameworks either consider conveying particular frameworks with thick transmitterrecipient connects or expound disconnected preparing strategy, that pieces quick sending and debilitates framework power. inside the paper, we tend to investigate to examination a novel finegrained constant adjustment free gadget free uninvolved human movement by means of physical layer information, that is autonomous of indoor situations and wants no earlier alignment and customary profile. We tend to explore sensitivities of abundancy and segment to human movement, and see that area include is extra delicate to human movement, especially to moderate human movement. Going for light-weight and hearty gadget free detached human movement identification, we tend to create two novel and sensible plans: here and now arrived at the midpoint of difference proportion (SVR) and long haul found the middle value of change proportion (LVR). We tend to see framework style with business WLAN gadgets and evaluate it in common multipath-rich indoor situations. As showed inside the analyses, our approach can complete a high recognition rate and low false positive rate.
\end{abstract}

\section{Keywords: physical layer information; device-free passive; human motion detection}

\section{INTRODUCTION}

Gadget free latent human discovery could be a thriving innovation to identify regardless of whether people, with none electronic instrument, exist inside the space of interests. it's an expanding interest and holds bigger possibilities to a few security-and wellbeing basic applications together with trespasser identification, resources insurance, senior nursing, and so forth., wherever gadget connection is badly designed or perhaps not feasible. With fast advancement of remote local space organize strategy, it's capability to comprehend blessing indoor remote gadget free detached human identification. early indoor remote latent human location utilized available got flag quality pointer (RSSI) from mackintosh layer. RSSI might be a coarse-grained and lowdetermination include. at interims the indoor zone, remote flag any experiences productive or harming multipath debilitating, prompting RSSI recklessness [1, 2]. When some individual hinders an attempt of transmitter (TX) and beneficiary (RX), RSSI of a connection may lessening, increment, or possibly remain unaltered [3]. As of late, fluctuated analysts investigate usage of remote local space arrange PHY layer data to acknowledge indoor gadget free detached human movement identification 
[4- 6]. The channel state data (CSI) of multitransporter signals portrays multipath parts, and square measure touchy to changes of immediate, intelligent or dissipating signals. Contrasted and RSSI, CSI is famous to incredibly enhance differ and precision of inactive human movement identification. In any case, created dynamic frameworks ar still frail in quick preparing and quality to changes of climate itself.

They either think sending particular frameworks with thick transmitter-recipient hyperlinks or troublesome disconnected training ways. The past objectives talented, overly complex preparing and last upkeep, that is not fitting for blessing indoor things. The last acknowledges genuine time location upheld cluster, pre-alignment or static example. bundle and pre-adjustment still include long and debilitating training endeavors.. Light-weight inactive human development identification totally depends upon on a trendy profile, and identifies human development by assessing bleeding edge flag design with static sign example. However when indoor insignificant environmental modification happens, it is required to recalibrate static illustration, that is appropriate to temperamental private or office space and speaks to a challenge to human development distinguishing proof. Previously mentioned inconveniences hinder the event of real time latent human development location in blessing indoor outcomes. Upheld the over inspiration, throughout this paper, we for the most part have a tendency to basically investigate the way to use PHY layer data to execute a progressed indoor best-grained ongoing detached human discovery (FRID) that might be immediately conveyed, unprejudiced of indoor various circumstances and any diversion or re-alignment. Then, thinking about prominent and regular inevitabilities of building and private, we tend to concur that a minor low assortment of sets of transmitter and recipient, even one connection, are more sensible decision for indoor slim locales.

Consequently, we will perceive the gentle weight real time inactive human recognition bolstered one connection with none prealignment or a popular profile. Next, we have a tendency to defy the fundamental test that the best approach to catch continuous inert human development disclosure with no arrangement or static illustration. Once two or three TX and RX is sent, do of area is begun up. Normally, recognizable proof plot totally relies on persistent information oblige the float, and distinguishes qualification between adjoining parcels. In this way, abundant tests were done to get the impact of human development on genuine time pointers. Lamentably, we have a tendency to watch that plentifulness refinement of bordering bundles isn't generally sufficiently delicate to human development. Plentifulness is moreover connected with indoor circumstances and losing off pointedly with expanding of separation among human region and observable pathway. In order to accomplish precision discovery, adjustment is unavoidable. Subsequently, the second one endeavor is that element it truly is tricky adequate to human movement are frequently separated from neighboring parcels. Beside the sufficiency work, we for the most part have a tendency to watch that component include is in idea a considerable measure of touchy to human development, especially to slow human development. Be that as it may, because of its no longer practical to precisely stay and right synchronization mix-ups of Wi-Fi gadget and business remote NICs, the crude part information carries on particularly haphazardly amid the conceivable field. Amid this paper, we have a tendency to gather direct and usable component data with the guide of making utilization of a straight change on the crude CSI to dispose of the different arbitrary clamor. By 
masses of investigations, the fresh out of the box new component information extricated from abutting bundles is tried to be various effective to detect human movement. In order to comprehend situation autonomous inactive human development recognition, we tend to separate proportion of stages between bordering bundles as key capacity. On the off chance that the earth is static, the proportion must be same to one. Something else, once somebody moves in a checking place, the proportion is pretty much than one. To raise the gadget power, we will be slanted to apply the statute on a succession of bundles at some phase in nearby time windows. Inside the device outline, we will be slanted to create novel constant human movement identification plans in light of coefficient of variation of stages: brief day and age found the middle value of change proportion (SVR) and long term arrived at the midpoint of fluctuation proportion (LVR). The two plans is prominent to evacuate the alignment cost and helpful to quick organization. Our essential duties are sketched out as takes after:

We propose ongoing uninvolved human movement identification by means of PHY layer information with no alignment. We tend to exploit physical-layer channel highlights, considering worldly security and part affectability to human movement. FRID is worthy for quick arrangement, independent of indoor different situations and vigorous to condition changes. We execute indoor finegrained constant aloof human movement recognition framework utilizing area data of CSI from ware Wi-Fi gadget. We tend to create two novel ongoing identifying plans in light of coefficient of variety of transient stage. FRID will with progress battle the negative impacts of indoor multipath and work on a solitary correspondence interface. To the least difficult of our data, we tend to be the essential to exclusively use the WLAN-based stage information to acknowledge ongoing uninvolved human movement location without alignment.

Broad assessments of FRID are led in two run of the mill indoor situations. The investigation comes about exhibit that FRID can do acceptable execution that outflanks RSSI-based framework.

In the rest of this paper, the associated work can be explored in Section a couple of, and preparatory concerning detached human movement recognition is given in Section 3. In Section 4, we tend to detail the examination concerning an approach to accomplish ongoing detached human movement recognition without adjustment, and set forward a viable stage based component in the Section 5. At that point we tend to detail the created two kinds of ongoing detached human movement recognition in the Section 6. Area 7 assesses the execution of FRID in two situations. Segment 8 closes the paper.

\section{RELATED WORK}

Since Youssef et al. [7] presented the possibility of Device-Free Passive (DfP) confinement, DfP were apparently and profoundly upset. A gadget free detached confinement gadget alludes to being fit for find, tune and see elements without conveying any related device. The recognition factor, as the essential way, is a crucial crude that is required through a wide assortment of developing projects. In early time, a large portion of human development discovery frameworks abused reachable mark, RSSI. To conquer inadequacies of RSSI, a couple of analysts are investigating to accomplish indoor CSI-based DfP human development identification.

RSSI-based Detection: RSSI might be conveniently removed from $\mathrm{ZigBee}$ or $\mathrm{WiFi}$ gadgets. In reference [7], creators achieved uninvolved human movement discovery in view 
of exchanging normal and exchanging change of WLAN-essentially based RSSI. Next, as the execution of gadget [7] defiles in a certified circumstance, the makers proposed a Maximum Likelihood Estimator (MLE) to update the general execution of the DfP system in honest to goodness conditions [8]. RASID [9] separated RSSI works and took after a non-parametric framework specifically circumstances to also enhance the execution of revelation. In reference [10],creators looked into extraordinary interruption styles and proposed a joint interruption picking up learning of approach construct absolutely with respect to two or three interruption signs to decorate the execution of interruption location. Likewise, anther wellacknowledge RSS-chiefly based DfP area is the Radio Tomographic Imaging (RTI) [11]. Starting late, different on a very basic level based-RTI DfP revelation and confinement are progressed, nearby the vRTI [12], kRTI [13], dRTI [3]. Despite its available get admission to, RSSI is coarse-grained and fails to get the multipath occurs [1] in indoor circumstances. Most structures simply use thick passed on frameworks to hit upon human proximity, which needs parcels work and gadgets and expands the expense of utilizations. Furthermore, because of the impedance of multipath comes about, the general execution of based-RSSI frameworks is negative for human moderate movement and quick development.

CSI-based Detection: Towards additionally light-weight and legitimate structures, most recent works jumped into the PHY layer and abused CSI for DfP discovery. FIMD [14] finished uninvolved human movement through bunch approach upheld the eigenvalues of closeness lattice of Canadian Security Intelligence Service. At that point Z. Zhou administration [15] anticipated AN omnidirectional uninvolved human location framework through abuse PHY layer capacities to basically track the state of watching scope. J. Xiao et al. [6] offered a Pilot gadget contributing fleeting equalization ANd recurrence separateness of CSI and reconciliation an Anomaly Detection square to encourage the instrument free perform. In reference [5], creators anticipated a direct free latent movement identification framework that recognizes one among a shape human conduct. Government Communications Commission [16] investigated the connection among the changeability of exchanging human and furthermore the variation of CSI and achieved the gathering checking basically based at the Gray Verhulst Model. Cushions [4] removed to be had fragment records of WLAN CSIs and joined the plentifulness with the portion to enhance the precision and heartiness of DfP human discovery. To condense, RSSI's principal drawback hinders the unavoidable utility of DfP frameworks in rich multi-course conditions, e.G., indoor circumstances. On the inverse, our acknowledgment in this watches is a WiFi-based absolutely better grained DFPL. Nonetheless, tantamount works rely upon either a confounded tutoring methodology or amassing masses of bundles to group, both of which result in diminished accommodation and materialness of human movement location. Not the same as past works, FRID takes advantage of stage capacity of CSIs and understands a lightweight real time detached human movement identification, that may suitably find human development with dynamic pace.

\section{ARCHITECTURE}

In this segment, we tend to exhibit the structure of FIMD close by configuration requesting circumstances. FIMD is a framework that endeavors the fitting choices of CSI from business NICs to give movement discovery. In far reaching, narrowband impedance at 2.Four ghz is unavoidable in a completely checked zone 
of indoor putting. In this manner, inside the nearness of narrowband impedance, the way to separate fitting alternatives from CSI for making sense of sign styles in static/dynamic conditions is that the underlying test we need to overcome. Channel State insights (CSI) is a reality that gauges the channel by method for speaking to the channel places of a discussion interface. Many specifically, CSI abuses the channel status when a RF sign engenders over more than one subcarriers. Naturally, CSI can show off absolutely exclusive options underneath static/dynamic environments. Indeed, even that CSI will differentially speak to the standard/dynamic examples; there need to in any case exist counterfeit recognition. For instance, the false caution energize can ascend because of the expanding substantial amount of info data. Additionally, the nearness of commotion underneath the mixed impact of, for instance, dispersing, blurring, and control rot with remove in gathered CSI tests should prompt ignore location. The second venture we need to attempt is the way to fittingly find a development event with limited confused. Additionally, from the state of mind of discussion proficiency, APs can transmission

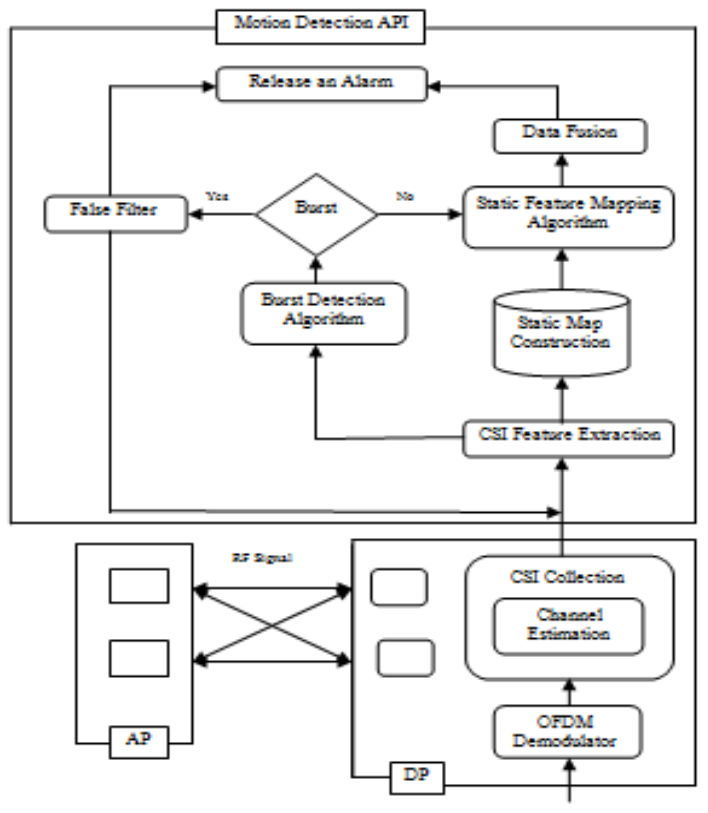

Fig. 1: System Architecture

Quality that enables us to most extreme the throughput. Nonetheless, RSS-based absolutely technique experiences this quality change and causes corrupting location exactness. The way to hold the recognition potential underneath alteration condition remains a third test in our works of art.

We initially depict the general innovative and judicious of FIMD as demonstrated in Fig. 1. FIMD comprises of three useful segments: inspire admission to factors (APs), distinguishing factors (DPs), and FIMD server. The APs can channel reference point messages over radio recurrence (RF) hyperlink. The DPs help the CSI arrangement common sense with the guide of transmitting RF sign. FIMD server at that point finishes the entire recognition strategy on-line. The APs and DPs are situated inside the space of pastime and unbroken work area bound all through the whole identification amount. In our putting, there are numerous sets of APs and DPs, each readied with two or three radio wires. Maintained these IEEE 802.11n requirements, not any more imperative gear ask for on each APs and DPs. Upon incidentally tolerating the OFDM flag message from APs, DPs would first have the capacity to accumulate the unrefined CSI worth inside the channel estimation piece. Specifically, let $\mathrm{x}$ be the transmitted vectors at APs, y be relating gotten vectors at DPs, severally. By then fine-grained CSI - channel get over all subcarriers at the PHY layer - are every now and again assessed as takes after:

\section{IV.REAL-TIME HUMAN MOTION DETECTION}

The execution of alignment based techniques like site-overview, pre-adjustment, building ordinary profile, and so on., might be enormously loaded with changes of condition 
itself and areas of furniture, that are unsatisfactory for cutting edge and variable circumstances. Furthermore, therefore, inside the paper, we tend to attempt and comprehend day and age gadget free inactive human movement identification with none action. We tend to benefit of steady of variety for recognizing sudden changes inside the CSI area for a Wi-Fi interface. Let $\delta \mathrm{i}, \mathrm{j} \Delta \mathrm{T}$ be the coefficient of variety for the jth transmitter-beneficiary radio wire combine and the ith OFDM subcarrier for the $\Delta \mathrm{T}$ time window. In order to discover worldly steady of variety transforms, we track $\delta$ $\mathrm{i}, \mathrm{j} \Delta \mathrm{T}$ over a fleeting window $\Delta \mathrm{T}$ and a longlasting time window $\Delta \mathrm{LT}$, and diagram two sorts of plans: here and now arrived at the midpoint of fluctuation proportion (SVR) and long haul found the middle value of difference proportion (LVR), allowing North American nation to check the different transient insights of the Wi$\mathrm{Fi}$ interface. The short window will repeat current state and be helpful to discover unexpected unusual occasion [12]. The long haul window speaks to a steady state, and influences FRID to void the re-alignment. Temporal variance ratio may be a relative metric that's not full of numerous environment conditions. We have a tendency to outline short-term averaged variance ratio (SVR) of section as:

$\mathrm{RSVR}=1 \mathrm{nn} \sum \mathrm{i}=1|\delta \mathrm{i} \Delta \mathrm{T} \delta \mathrm{i} \Delta \mathrm{T}-1| \ldots . .(1)$

SVR could be a light-weight process scheme that quickly observes abrupt dynamic changes of the environment. We tend to take note of that our window-based fluctuation proportion system varies from the past strategies [5, 9]. The past light-weight human movement location are frequently come through by contrasting late window-based element estimations with estimations made all through a static action sum once nobody is getting the universe of intrigue. Be that as it may, on account of alterable indoor situations, the static example is in danger of disappointment, and re-alignment is normally dead. Then again, the SVR exclusively speaks to late flag changes between neighboring windows. Once the human is consistently moving, the flag changes between neighboring windows could likewise be comparable, and furthermore the SVR may neglect to watch the human movement. Subsequently, to catch the conduct of remote connections once the main part of estimations are conceivable made though the earth is static and watch ceaseless flag transforms, we tend to apply the steady of variety of part on a long-run time window. We have a tendency to outline long-term averaged variance ration (LVR) of part as: $\mathrm{RLVR}=1 \mathrm{nn} \sum \mathrm{i}=1|\delta \mathrm{i} \Delta \mathrm{T} \delta \mathrm{i} \Delta \mathrm{T}-1| \ldots . .(2)$ where $\mathrm{n}$ is the number of subcarriers, $\delta \mathrm{i} \Delta$ Tis the coefficient of variation of phase of $i^{\text {th }}$ subcarrier in time intervals $\Delta \mathrm{T}, \delta \mathrm{i} \Delta \mathrm{LT}$ is the coefficient of variation of phase of $i^{\text {th }}$ subcarrier in a long time intervals $\Delta \mathrm{LT}$. $\delta \mathrm{i} \Delta \mathrm{LT}$ Tan be obtained from the coefficient of variation of phase of $i^{\text {th }}$ subcarrier when the number of corresponding continuous normal SVR is $\mathrm{N}$. When no one moves, the RLVR also falls in a confidence interval $\left(1-\frac{Z \alpha}{2} * \lambda\right)<R L V R<\left(1+\frac{Z \alpha}{2} * \lambda\right)$,

where $\lambda$ is the experiential standard variation of RLVR in static environment. The RLVR is calculated based on a stable state when the RSVR is within a normal range for a long time. The RLVR is efficient to identify an abnormal state and a stable state.

The human movement is judged by joining SVR with LVR. We have a tendency to initially utilize the SVR to find regardless of whether relate gatecrasher moves into the checking space.

As said over, it's mistaken to gather regardless of whether the contestant extra strolls into or leaves from the checking space. Subsequently, we will use the LVR to follow regardless of whether the individual strolls constantly at interims the observing space. On 
the off chance that the participant doesn't appear inside the checking space, the $\delta$ I $\Delta \mathrm{LT}$ will be refresh with late $\delta \mathrm{I} \Delta \mathrm{T}$. Inside the case wherever there is additionally different recieving wire sets, we tend to take the mass vote between radio wire combines over the term window to go to a choice if a man's movement occasion has happened. Extra particularly, once a collector radio wire identifies an irregular occasion, we tend to check the unusual discoveries for all the beneficiary recieving wires over the RSVR and RLVR. For a two $\times$ two MIMO transmitters and collector, this can mean registering a greater part vote more than eight estimations. At the point when the majority of the recipient recieving wires see human movement, we tend to surmise that somebody is moving between the transmitter and accordingly the beneficiary. we'll demonstrate that this greater part vote approach enhances the execution of our indicator by diminishing false alerts and uncomprehensible detections. we have a tendency to decrease the warning rate more by combining temporally shut detections along.

$$
\mathrm{H}=\frac{\mathrm{y}}{\mathrm{x}} \ldots \ldots . .(4)
$$

Starting there ahead, FIMD server will import the CSI estimation accumulated by the DPs and start the area convenience. There exist five urgent modules managing the server, including: CSI Feature Extraction, Burst Detection, StaticMap Construction, False Alert Filter, and Data Fusion. In the consigned CSI Feature Extraction module, foul CSI made from 30 social event's varying subcarriers will be first masterminded. Instinctually, channel status information CSI will display differential attributes in static and dynamic conditions. We lead preparatory analyses in run of the mill indoor situations to approve this instinct. We prevail with regards to misusing the normal for CSI which uncovers ordinary and movement conduct from multiple points of view in which. A most eigenvalue over window is utilized to speak to the element cost respect conventional or movement conduct. Next, the Burst Detection module continues running on the arranged CSIbased component cost dataset over different arrangements of associations severally. For burst disclosure, the association standing is inspected using a thickness based DBSCAN course of action run the show. The represent can break down the part cost inside the dataset of each associate with give packs. If the concentrations in an exceedingly dataset have a place with one gathering, the relevant standing is thought to be static. In capability, if there exists more than one bundle inside the specific dataset, it ought to be a dynamic remaining in perspective of development direct. Since there might exist false area, additional examination ought to be done to help the general distinguishing proof execution. As demonstrated by the hidden got occurs as a result of Burst Detection, we tend to deliver 2 cases refinement: 1) forewarning Filter: using a reasonable windowing rule, we will disconnect out the false area that mistakenly make a burst alarm once no development shows up; 2) information Fusion: even once no burst has been perceived all through the basic burst acknowledgment territory, there could exist some missing cases. Thusly, we have a tendency to enhance the recognizable proof accuracy by including this information Fusion module and revive the static component of arranged CSI. In what tails, we will detail this masterminded structure in A to an awesome degree partitioned and-beat way.

\section{METHODOLOGY}

In this segment, we tend to have a tendency to clarify the outline wording of FIMD. The technique of this CSI-based movement discovery approach is typically relaxed into 5 parts in advance with the relating modules presented in previous section III.

\section{A. CSI Feature Extraction}


As per our adjustment of chipset firmware, the foul CSIs are separated into 30 packages each with 2 subcarriers. The $\mathrm{N}=30$ groups CSI views can be granted as

$\mathrm{H}=\left[\mathrm{H}_{1}, \mathrm{H}_{2} \ldots . \mathrm{H}_{\mathrm{i}}, \ldots \ldots \mathrm{H}_{\mathrm{N}}\right]^{\mathrm{T}}, \mathrm{i} \in[1,30], \ldots \ldots(5)$

where each subcarrier $H_{i}$ is defined as

$$
\mathrm{H}_{\mathrm{i}}=\left|\mathrm{H}_{\mathrm{i}}\right| \mathrm{e}^{\mathrm{j} \sin \left\{\angle \mathrm{H}_{\mathrm{i}}\right\}}, \ldots . .(6)
$$

where $\left|H_{i}\right|$ is the amplitude response and $\angle H$ is the phase response of the $i_{t h}$ subcarrier.

The main central module - CSI Feature Extraction is an essential of the subsequent modules. The center arrangement of this module is to strategy the thirty bunch Canadian Security Intelligence Service data got from different DPs, and investigate the attributes of CSI that recognize the flag designs underneath static or dynamic conditions.

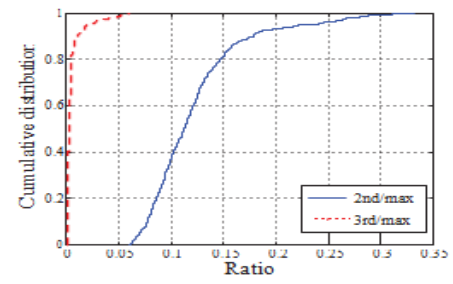

Fig. 2: CSI Feature Extraction

In particular, we have a tendency to separate the CSI dynamic example from CSI stationary example because of the development of elements.

To this end, we first process consistent CSIs beginning from $\mathrm{Hk}$ once again a sliding window $\mathrm{W}$. Given a sliding window $\mathrm{W}$ with length $\mathrm{n}$, CSIs can be communicated $\operatorname{asH}=\left[\mathrm{H}_{\mathrm{k}}, \mathrm{H}_{\mathrm{k}+1}, \ldots . \mathrm{H}_{\mathrm{k}+\mathrm{n}}\right], \ldots . .(7)$

Next, we want to spot the properties of CSI that reflects static/dynamic signal patterns. so as to get the correlation issue between every column of $\mathrm{H}$, we have a over the $\mathrm{n}$ consecutive packets asC tendency to generate a n-by-n squarematrix $\mathrm{C}=\left[\begin{array}{ccc}\mathrm{C}(\mathrm{i}, \mathrm{i}) & \cdots & \mathrm{C}(\mathrm{i}, \mathrm{i}+\mathrm{n}) \\ \vdots & \ddots & \vdots \\ \mathrm{C}(\mathrm{i}+\mathrm{n}, \mathrm{i}) & \cdots & \mathrm{C}(\mathrm{i}+\mathrm{n}, \mathrm{i}+\mathrm{n})\end{array}\right]$

where each element $\mathrm{C}(i, j)$ in the matrix $\mathrm{C}$ is the correlation ratio between the $\mathrm{H}_{i}$ and $\mathrm{H}_{j}$ as

$\mathrm{C}(\mathrm{i}, \mathrm{j})=\operatorname{corr}\left(\mathrm{H}_{\mathrm{i}}, \mathrm{H}_{\mathrm{j}}\right)$

The estimation of corner to corner passages in network $\mathrm{C}$ is leveled with to 1 . In our technique, we duplicate a scalar $\lambda$ to acquire the eigenvector eigen of lattice C. In this way, the CSI include extraction issue is equal to finding the most extreme eigenvalue of this eigenvector after standardization

The feature value associated with CSI is defined as $\mathrm{V}$,

$$
\mathrm{V}=\max (\operatorname{eigen}(\mathrm{C}) / \mathrm{n}) \ldots \ldots(10)
$$

where $n$ is the sliding window length that constraints the column number of matrix $\mathrm{C}$.

In the event that all the eigenvalue of every segment unit the same all in all, the relating maximumeigen $(\mathrm{C})$ equivalents to 1 though the rest unit zero. Along these lines, with higher connection between every section in $\mathrm{H}$, the flag will show promote apparently to be static. Conversely, if the eigenvalue all of a sudden diminishing to alittle esteem, the lower relationship would perhaps show scholarly degree happen rence of movement. we tend to have a tendency to directed preparatory analyses for substantiating the anticipated component extraction approach. Ordinarily, most|the utmost|the most $\}$ and second most eigenvalues unit expansive while from the third one, the eigenvalue turns out to be close to nothing and unit considered immaterial appeared in 


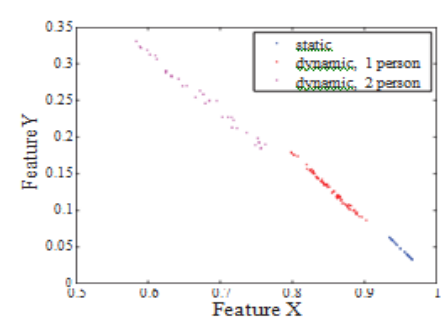

Fig. 3: CSI Features in Static/Dynamic Environments

Fig. 3. We plot the most extreme and second greatest eigenvalues indicated as highlight ( $\mathrm{x}-$ pivot) and highlight (y-hub) on a2dimensionalFig. 3, individually. There are three fundamental perceptions from the figure: 1) The eigenvalue in static status is most extreme and drawing closer to $1 ; 2$ ) The eigenvalue will wind up noticeably littler in the dynamic conditions; 3 ) If more individuals introduced in the area of intrigue, the eigenvalue will additionally diminish because of higher change.

Obtained from CSI-based association network C, eigenvalue $\mathrm{V}$ is been feature lying on two striking points of interest. To begin with, such eigenvalue $\mathrm{V}$ is self-governing with control. RSS-based approach is known to be helpless to transmitted power at the APs, and in this way requires additional APs supplement. On the other hand, CSI-construct eigenvalue depends in light of connection over various gatherings CSIs and immaterial to control evolving. Second, this component esteem is strong to narrowband impedance at $2.4 \mathrm{GHz}$.

\section{B. Static Profile Construction}

FIMD's Static Profile Construction module addresses the stationary banner plans in the watched an area. In our FIMD structure, this is an optional module just if detached getting ready is available and basic. It should be seen that we won't use this module in our gathering based revelation, however for the relationship with $\mathrm{RSS}$, this module is used as a piece of the
RASID-like approach.

Reasonably like late work [4] that utilizations non-parametric piece thickness estimation of RSS esteem after some time, we at that point propose to use the more worldly stable metric CSI and develop a static element profile. When all is said in done, the development procedure should investigate the recurrence decent variety of CSI that speaks to the conspicuous static example frequencies over different subcarriers. Thusly, rather than utilizing the coarse RSS characterized in the evaluated thickness work [4], this module inputs the stationary CSI-based component esteems created from the Feature Extraction Module.

\section{Burst Detection}

The key module of our FIMD system is Burst Detection, which assumes a critical part in the location procedure. It screens the events of CSI difference because of movement occasions

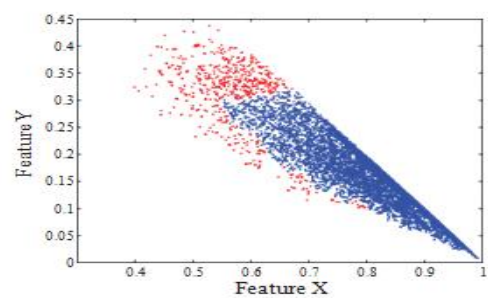

Fig 4: DBSCAN Clustering Results

during our estimation period. Specifically, Burst Detection sees movement location as an example acknowledgment issue, as opposed to a mark coordinating issue. It depends on the way that the examples of movement occasions are essentially abnormal, and stray from the static ones. With the end goal that the burst CSI designs are considered a conceivable movement activity. In this way, we require a powerful calculation to characterize the CSI designs and decide the "burst" movement event. Thickness based arrangement calculation DBSACN [7] is a solid match for Burst Detection in view of two great highlights: (1)no earlier learning of the 
quantities of groups is required (2)discovery of bunches with subjective shape.

There are two information parameters in our calculation includinge (eps) - the sweep that delimitate the area region of a factor, signified as $\mathrm{N}$ (p) minP ts - least number of components that should exist inside the $\varepsilon$-neighborhood factors,

The key course of action of the DBSCAN group pick is that, for each reason in an amazingly assembling, $\varepsilon$-neighborhood needs to join at any rate additional than the minP ts. That is, the thickness inside the $\varepsilon$-neighborhood needs to outflank some predefined edge. Given a specific CSI-on an exceptionally fundamental level based trademark worth dataset of a RF hyperlink among AP and removed individual, the DBSCAN gather control consents to the consequent benchmarks:

- Principle 1: Each group contains no less than one component esteem $\mathrm{Vi}$ as center point $\mathrm{p}$ that the span of $\mathrm{N}(\mathrm{p})$ is at any rate minP ts.

- Principle 2: Given any two component esteems V1and V2with size of $\varepsilon$ neighborhood more prominent than $\operatorname{minP}$ ts, at that point V1and V2are in a similar group.

- Principle 3: In the event that element esteem Vi has size of $\varepsilon$-neighborhood not as much as minP ts, and no center point is contained in $\mathrm{N}(\mathrm{p})$, at that point $\mathrm{Vi}$ is an anomaly.

Keeping in mind the end goal to talk about whether an arrangement of focuses is sufficiently comparative to be viewed as a group, we require a separation measure $\operatorname{Dist}(\mathrm{Vi}, \mathrm{V} \mathrm{j})$ which tells how far focuses $\mathrm{Vi}$ and $\mathrm{Vj}$ are. In our calculation, we apply Euclidian recipe to gauge $\operatorname{Dist}(\mathrm{Vi}, \mathrm{V}$ j) as takes after:

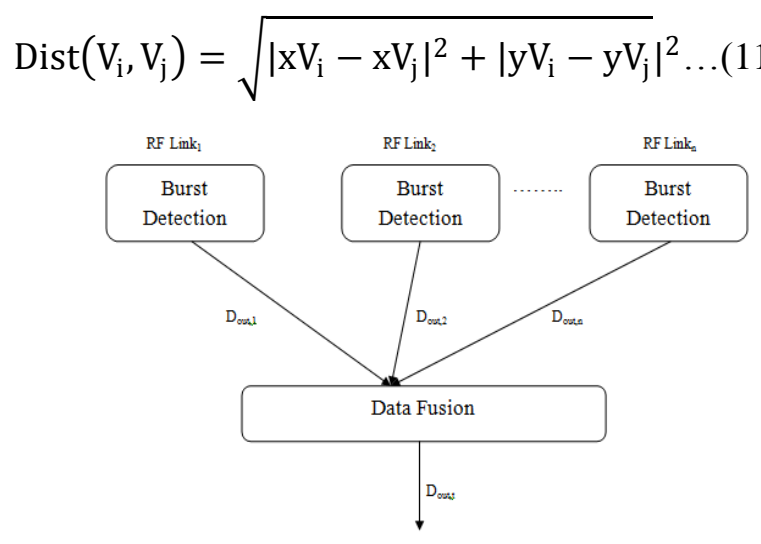

Fig. 5: Data Fusion over Multiple RF Links

The measure of feature regards delegated to a particular cluster inside a sliding window is extra than an edge $\eta$, the nation is thought to be static. Something different, it's miles named a "Burst" country. Fig. 4 serves for example to reveal how DBSCAN is fit for discover the development occasion of moving toward component cost dataset. As implied in Sec. IV$\mathrm{A}$, we make the most eigenvalue and the second most noteworthy eigenvalue as feature regards. We relate each trademark charge as a factor on a 2-dimensional thickness chooses, and the outcomes of the gathering appraisal in static/dynamic are shown in Fig. 4.

As once in the past stated, identification exactness is the essential plan reason for FIMD framework; we need to diminish the blunders that without a doubt came to pass for in the entire location framework. So we additionally perform two lessons of plans over the Burst Detection results to determine the phony caution and ignore location as takes after:

\section{False Alarm Filter}

From the point of view of enhancing location capacity, a basic Burst Detection might be lacking. Rather, So we moreover perform two lessons of plans over the Burst Detection results to decide the fake alert and overlook area as 
takes after. Here, we apply a straightforward windowing strategy.

Seen from the observational examination, a solitary advancement delineation dependably keeps going a succinct period while enduring reliable gatherings. With the genuine target that the dynamic case can be settled from CSI-based segment respect over a particular sliding window $\mathrm{W}$, too from the ones of every a brief moment to the opposite side and right of $\mathrm{W}$. In light of the windowing channel, we move the window to the opposite side neighbor and right neighbor and figure the taking a gander at include respect. On the off chance that the segment respect in window is kept from the flanking ones, by then the "burst" illustration made from Burst Detection module can be settled as a false area and filtered through.

\section{E. Data Fusion}

Another wellspring of mistaken location is known to be miss identification. That is, for miss discovery, we play out extra strides to diminish the miss location as few as would be prudent. Beforehand, each single RF connect creates an underlying recognition comes about in light of Burst Detection calculation, which the yield is characterized into either typical static or anomaly dynamic.

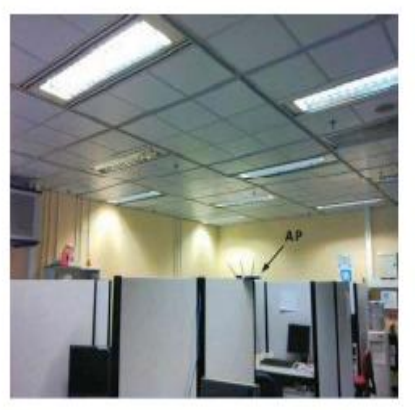

Fig. 6: Research Laboratory

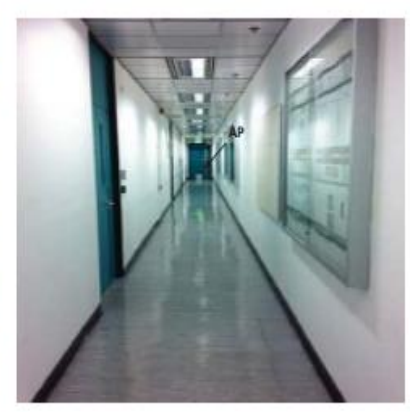

Fig. 7: Testbed2:Corridor

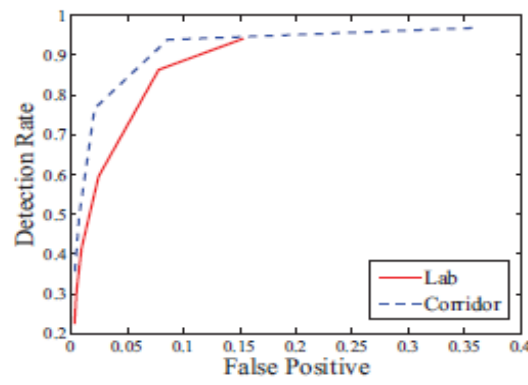

Fig. 8: ROC in Two Testbeds

Missed Filter abuses data mix framework over multi-interface CSIs, which synergistically planning the CSIs from various associates with convey broad information about an acknowledgment event. This results in a diminished false negative revelation rate over single-interface approach. Fig. 5 exhibits different RF joins contributing their decisions whether a development has happened or not to a fuser.

\section{PERFORMANCE EVALUATION}

1) Evaluation Metric: We set up the running with metric to get to the execution of the proposed FIMD framework: True Positive (TP) Rate: TP rate recommends the likelihood that an advancement occasion is legitimately seen.

2) Experimental Results:: First, we delineate a Receiver Operating Characteristic (ROC) contort that graphically translates the affirmation execution inside observing false caution. ROC bend can unequivocally demonstrate the tradeoff between the FP rate (Xfocus) and TP rate (Y-turn). Here, we utilize DR to address the TP rate, which measures the 


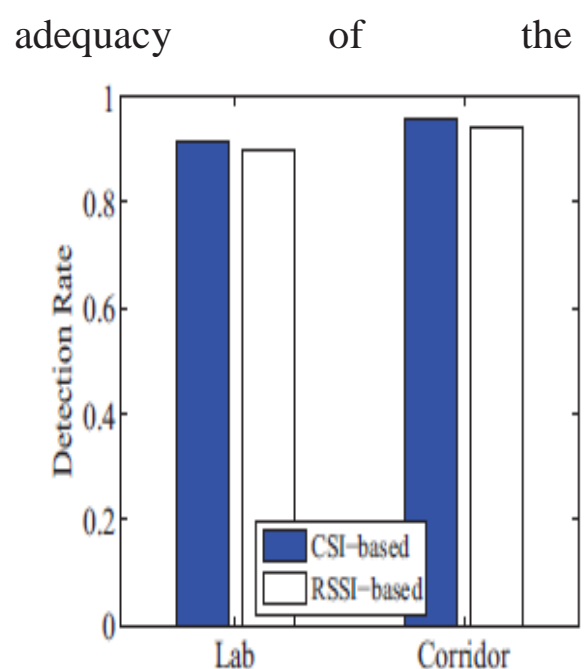

Fig. 9: CSI-based Feature vs. RSS-based Feature

system according to the following Equ. 12,

$$
D R=\frac{T P}{(T P+F N)} * 100 \% \ldots . .
$$

Fig. 8 gives the MDR rate regard to false alert rate in two testbeds. In Lab, for a FP rate not decisively or equivalent to $1 \%$ the divulgence rate would be more huge than $70 \%$, and for a FP rate more basic than $14 \%$ the ID rate would be more unmistakable than $90 \%$. Likewise, the ROC bend in Corridor demonstrates that revelation rate would be more unmistakable than $90 \%$ when FP rate is around $9 \%$.

Examination with RSS-based: So far, we have been concentrating on the execution of the proposed CSI-based FIMD structure. To assess the gainful get of CSI-based segment over RSSbased segment, we look at it against the most material RSS-based improvement affirmation structure RASID. RASID is a momentous RSSbased without device lethargic affirmation structure which incorporates an isolated preparing stage and a web checking stage. It use standard deviation (SD) of RSS as the section approximates it course with a section work. For a sensible association, we keep the entire RASID disclosure process and just supplant the RSSbased part with proposed CSI-based segment. Where basically the best eigenvalue disengaged from CSI relationship over a sliding window. We execute RASID on FIMD server in both testbeds and set the sliding window length to be 10 as appeared in Fig. 9. The length of resuscitate window is settled to be 30 . From Fig. 9, we can watch that CSI-based part scarcely beats the RSS-based one with the genuine goal of advancement region in perspective of better normal security. In rundown, CSI-based part can furnish better conspicuous verification execution separating and the assistant in context of RSSI.

VII. CONCLUSIONS AND FUTURE WORK

In the paper, we have a tendency to propose novel light-weight and timeframe detached human movement identification. With the quick advancement of remote gadget free detached restriction, indoor fine-grained latent human recognition has been wide looked into. Timeframe detached human identification are frequently apace sent and wants no gigantic site study. In addition, human movement wills unmistakably alteration the piece of multipath signals. Henceforth, we have a tendency to accomplish a FRID framework, fine-grained timeframe latent human movement location through PHY layer part information. To welcome the timeframe human movement recognition, we have a tendency to create 2 plans: short arrived at the midpoint of difference quantitative connection (SVR) and semiperpetual found the middle value of fluctuation quantitative connection (LVR). Shifted tests have very much attempted that the FRID framework can do superior, especially for moderate human movement. Inside the future, we'll investigate to utilize a considerable measure of cutting edge methods to support the execution of latent human movement recognition by means of the aggregate CSI 
information.

\section{REFERENCES}

1. N. Patwari,"Robust Location Distinction using Temporal Link Signa-tures," in Proc. of ACM MobiCom, 2007.

2. M. Youssef, M. Mah, A. Agrawala,"Challenges: Device-free Passive Localization for Wireless Environments," in Proc. of ACM MobiCom, 2007.

3. M. Moussa and M. Youssef,"Smart Devices for Smart Environments: Device-free Passive Detection in Real Environments," in Proc. of IEEEPerCom Workshops, 2009.

4. A.E. Kosba, A. Saeed, M. Youssef,"RASID: A Robust WLAN Device-free Passive Motion Detection System," in Proc. of IEEE PerCom, 2012.

5. J. Zhang, M.H. Firooz, N. Patwari, S.K. Kasera,"Advancing Wireless Link Signatures for Location Distinction," inProc. of ACM MobiCom, 2008.

6. J. Zhang, S.K. Kasera, N. Patwari, P. Rai,"Distinguishing Locations Across PerimetersUsingWirelessLink

Measurements," in Proc. of IEEEINFOCOM, 2011.

7. M. Ester, H.P. Kriegel, J. Sander, X. Xu, "A density-based algorithm for discovering clusters in large spatial databases with noise," in Proc.of the Second International Conference on Knowledge Discovery and Data Mining (KDD), 1996.

8. M. Wallbaum, S. Diepolder,"A Motion Detection Scheme For Wireless LAN Stations," in Proc. of ICMU, 2006.

9. R. S. Moore, R. Howard, P. Kuksa, and R. P.Martin, "A Geometric Approach to Device-Free Motion Localization Using Signal Strength," in Technical Report,
Rutgers University, 2010.

10. J. Yang, Y. Ge, H. Xiong, Y.Y. Chen, and H.B. Liu, "Performing joint learning for passive intrusion detection in pervasive wireless environments," in Proc. of IEEE INFOCOM, 2010.

11. D.J. Kim, B. Prabhakaran, "Motion fault detection and isolation in Body Sensor Networks," in Proc. of IEEE PerCom, 2011.

12. Zhang, J. Ma, Q. Chen, and L.M. Ni, “An RF-Based System for Tracking Transceiver-Free Objects," in Proc. of IEEE PerCom, 2007.

13. Zhang, Y.H. Liu, L.M. Ni,"RASS: A real-time, accurate and scalable system for tracking transceiver-free objects," in Proc. of IEEE PerCom, 2011.

14. A. Bhartia, Y. Chen, S. Rallapalli, and L. Qiu, "Harnessing Frequency Diversity in Wi-Fi Networks," in Proc. of $A C M$ MobiCom, 2011.

15. K. Wu, J. Xiao, Y. Yi, and Lionel M. Ni, "FILA:Fine-grainedIndoorLocalization," in Proc. of IEEE INFOCOM, 2012.

16. J. Lv, W. Yang, L. Gong, D. Man, and X. $\mathrm{Du}$, "Robust WLANBased Indoor FineGrained Intrusion Detection," presented at the 2016 IEEE Global Communications Conference (GLOBECOM), Washington, DC, USA,

17. J. Lv, W. Yang, D. Man, X. Du, M. Yu, and M. Guizani, "Wii: Device-free Passive Identity Identification via WiFi Signals," presented at the 2017 IEEE GlobalCommunicationConference(GLO BECOM), Singapore, 2017.

18. L. Wei, R. Q. Hu, Y. Qian, and G. Wu, "Enabledevice-to-devicecommunications underlayingcellular networks: challenges and research aspects," IEEE Communications Magazine, vol. 52, pp.90-96,2014. 